\title{
Ocular infection with an avian trematode (Philophthalmus sp)
}

\author{
R D K Rajapakse ${ }^{1}$, K M T N Wijerathne ${ }^{2}$ and M de S Wijesundera ${ }^{2}$
}

(Index words: Philophthalmus, human philophthalmosis, trematode zoonoses, ocular parasites, eye fluke)

\section{Introduction}

Adult trematodes of the genus Philophthalmus that parasitize the conjunctival sac of birds can cause accidental human infection. Human philophthalmosis is rare, but isolated cases have been reported from several countries including Sri Lanka [1-6]. Adult flukes lay eggs that hatch on contact with water and develop in snail hosts. The emerging cercariae encyst on animate or inanimate objects in water infecting avian definitive hosts through ingestion, and then migrate to the eye. However, direct ocular infection can occur [7], and is the common route of infection in humans. We report the third case of philophthalmosis in Sri Lanka, and probably the first report of an indigenous infection.

\section{Case report}

A 32-year old housewife from Hingurakgoda presented at the Polonnaruwa General Hospital with redness, irritation and a dull pain in her left eye of two weeks duration. There was no history of trauma. No visual impairment or eye discharge was detected. Slit lamp examination showed dilated conjunctival and episcleral blood vessels at the superior limbus resembling typical episcleritis. Closer examination showed a small 'worm', about $1 \mathrm{~mm}$ in size, moving rapidly under the conjunctiva in the episcleral space at the superior limbus. The cornea, anterior chamber, lens, vitreous and fundus were normal. The 'worm' was extracted through a conjunctival incision and transported in normal saline for identification.

The patient lived with her husband and two children. Five years ago she had lived in Bahrain for two years, but had not traveled overseas since then. Their source of drinking water was a well but she bathed in an irrigation canal. She was a strict vegetarian for the past three years. No other family members had a similar illness. The patient was treated with topical steroids and antibiotics for one week. The symptoms subsided within a week and the steroid was tailed off. She remained symptom free on review at three months.

\section{Parasite morphology}

The parasite was a live specimen, and was fixed in formalin and stained with aceto-carmine. It was a complete adult trematode, spindle shaped, measuring $1.5 \times 0.525$ $\mathrm{mm}$ with an oral and a large ventral sucker. Due to poor relaxation prior to fixing the ventral sucker was damaged. The well developed spherical pharynx abutted the oral sucker (Figure 1). The oral to ventral sucker ratio was 1 : 1.66. The uterus had eggs containing miracidia larvae with eyespots (Figure 2) indicating that it was a mature trematode. The testes, uterus and vitelleria were indistinct for morphological study. It was identified as a species of Philophthalmus.

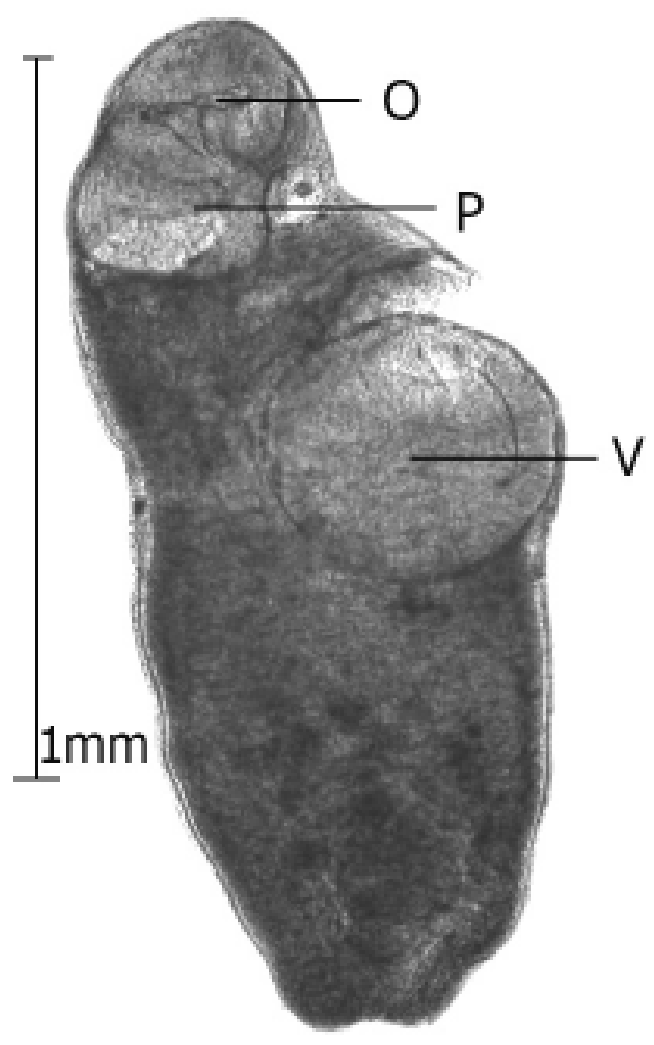

Figure 1. Adult Philopthalmus recovered from the eye showing the oral sucker $(O)$, the pharynx $(P)$ and the ventral sucker (V) (Aceto-carmine stain).

${ }^{1}$ General Hospital, Polonnaruwa and ${ }^{2}$ Department of Parasitology, Faculty of Medicine, University of Peradeniya, Sri Lanka.

Correspondence: MdeSW, e-mail <wijem@sltnet.lk>. Received 11 June 2009 and accepted 8 August 2009. Competing interests: none declared. 


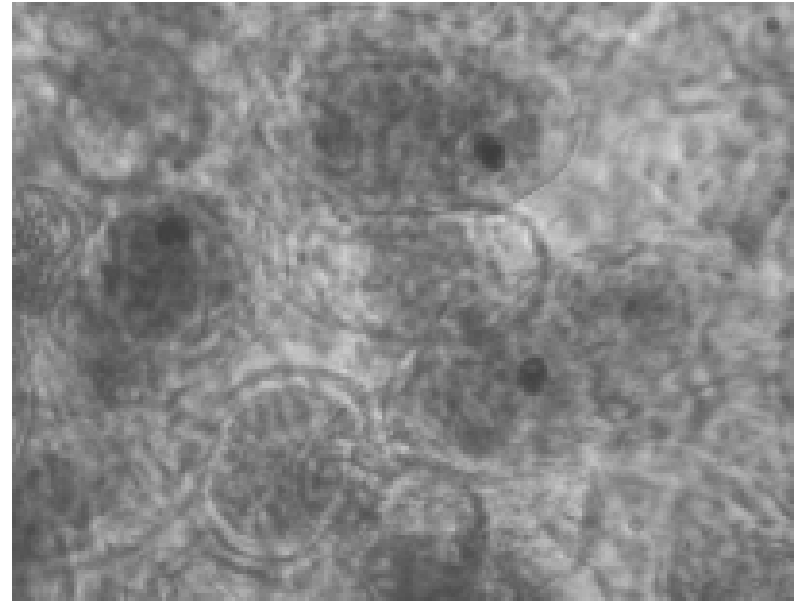

Figure 1. Eggs in the uterus showing miracidia with eyespots (magnification $\times 1000$ ).

\section{Discussion}

Over 50 species have been described in the genus Philophthalmus, but only 10 have been validated. Of the human infections only the Mexican infection has been conclusively identified to species level as $P$. lacrimosus [5]. Of the two previous case reports from Sri Lanka, the first was in an Indian resident who frequently travelled to South India and infection was presumed to have been acquired in India [1]. The second case was diagnosed in Berlin in a Sri Lankan adult male who had arrived in Germany three months previously from Kayts, Northern Sri Lanka [2]. Unfortunately his ethnicity, occupation or travel were not noted. Hence our case is probably the first report of indigenous infection.

In all human infections other than a case report from Thailand [6], infection was probably due to direct entry of trematode larvae during bathing or swimming in infected fresh water. However, infection through ingestion of metacercariae is possible. Infection is not known to cause serious illness in either humans or the avian natural hosts. However, in a recent prospective study on granulomatous anterior uveitis in 113 children in Tamil Nadu and Kerala, several lesions have been attributed to infection with Philophthalmus [8]. The authors have recognised philophthalmosis as an endemic water-borne parasitic disease, acquired by exposure to freshwater in village ponds and rivers, and highlighted the need for proper management in order to prevent complications. The irrigation canals and rivers in the dry zone of Sri Lanka could be a source of infection. Studies on Philophthalmus larval infection in fresh water snails should be undertaken to ascertain the parasitic species present in Sri Lanka.

\section{Acknowledgements}

We thank Mr. R. L. A. R. Ranaweera for technical assistance, Dr. Charaka Dasanayake for photomicrography and Ms. Claire Hemachandra for translating the German publication.

\section{References}

1. Dissanaike AS, Bilimoria DP. On an infection of a human eye with Philophthalmus sp. in Ceylon. Journal of Helminthology 1958; 32: 115-8.

2. Kalthoff H, Janitschke K, Mravak S, et al. Ein ausgereifter saugwurm der gattung Philopthalmus unter der bindehaut des menschen. Klin Monatsbi Augenheilkd 1981; 179: 373-5.

3. Mimori T, Hirai H, Kifune T, Inada K. Philophthalmus sp. (Trematoda) in a human eye. American Journal of Tropical Medicine and Hygiene 1982; 31: 859-61.

4. Lang Y, Weiss Y, Garzozi H, et al. A first instance of human philopthalmosis in Isreal. Journal of Helminthology 1993; 67: 107-11.

5. Lamothe-Argumedo R, Diaz-Camacho SP, Nawa Y. The first human case in Mexico of conjunctivitis caused by the avian parasite, Philophthalmus lacrimosus. Journal of Parasitology 2003; 89: 183-5.

6. Waikagul J, Dekumyoy P, Yoonuan T, Praevanit R. Conjunctival philophthalmosis: a case report in Thailand. American Journal of Tropical Medicine and Hygiene 2006; 74: 848-9.

7. Alicata JE, Ching HL. On the infection of birds and mammals with cercariae and metacercariae of the eye-fluke Philophthalmus. Journal of Parasitology 1960; 46 (Supp.): 16.

8. Rathinam, Sivakumar R, Usha KR, Rao NA. Presumed Trematode-induced granulomatous anterior uveitis: a newly recognized cause of intraocular inflammation in children from South India. American Journal of Ophthalmology 2002; 133: 773-9. 